\title{
Invariant Statistical Convergence of Sequences of Sets with respect to a Modulus Function
}

\author{
Nimet Pancaroglu and Fatih Nuray \\ Department of Mathematics, Afyon Kocatepe University, Afyonkarahisar, Turkey \\ Correspondence should be addressed to Fatih Nuray; fnuray@aku.edu.tr \\ Received 15 December 2013; Accepted 21 February 2014; Published 23 March 2014 \\ Academic Editor: Irena Rachůnková
}

Copyright (c) 2014 N. Pancaroglu and F. Nuray. This is an open access article distributed under the Creative Commons Attribution License, which permits unrestricted use, distribution, and reproduction in any medium, provided the original work is properly cited.

We introduce and study the concept of invariant convergence for sequences of sets with respect to modulus function $f$ and give some inclusion relations.

\section{Introduction}

The concept of statistical convergence for sequences of real numbers was introduced by Fast [1] and studied by Šalát [2] and others. Let $K \subseteq \mathbb{N}$ and $K_{n}=\{k \leq n: k \in K\}$. Then the natural density of $K$ is defined by $\delta(K)=\lim _{n} n^{-1}\left|K_{n}\right|$, if the limit exists, where $\left|K_{n}\right|$ denotes the cardinality of $K_{n}$.

A sequence $x=\left(x_{k}\right)$ complex numbers is said to be statistically convergent to $L$ if, for each $\varepsilon>0$,

$$
\lim _{n} \frac{1}{n}\left|\left\{k \leq n:\left|x_{k}-L\right| \geq \varepsilon\right\}\right|=0
$$

Convergence concept for sequences of set had been studied by Beer [3], Aubin and Frankowska [4], and Baronti and Papini [5]. The concept of statistical convergence of sequences of set was introduced by Nuray and Rhoades [6] in 2012. Ulusu and Nuray [7] introduced the concept of Wijsman lacunary statistical convergence of sequences of set. Similarly, the concepts of Wijsman invariant statistical and Wijsman lacunary invariant statistical convergence were introduced by Pancaroglu and Nuray [8] in 2013.

A function $f:[0, \infty) \rightarrow[0, \infty)$ is called a modulus, if

(1) $f(x)=0$ if and if only if $x=0$;

(2) $f(x+y) \leq f(x)+f(y)$;
(3) $f$ is increasing;

(4) $f$ is continuous from the right at 0 .

A modulus may be unbounded (e.g., $f(x)=x^{p}, 0<p<$ 1) or bounded (e.g., $f(x)=x /(x+1)$ ).

Modulus function was introduced by Nakano [9] in 1953. Ruckle [10] used in idea of modulus function $f$ to construct a class of FK spaces. Consider

$$
L(f)=\left\{x=\left(x_{k}\right): \sum_{k=1}^{\infty} f\left(\left|x_{k}\right|\right)<\infty\right\} .
$$

The space $L(f)$ is closely related to the space $\ell_{1}$ which is a $L(f)$ space with $f(x)=x$, for all real $x \geq 0$.

Maddox [11] defined the following spaces by using a modulus function $f$ :

$$
w_{0}(f)=\left\{x \in s: \lim _{n \rightarrow \infty} \frac{1}{n} \sum_{k=1}^{n} f\left(\left|x_{k}\right|\right)=0\right\},
$$

$$
w(f)=\left\{x \in s: \lim _{n \rightarrow \infty} \frac{1}{n} \sum_{k=1}^{n} f\left(\left|x_{k}-L\right|\right)=0 \text { for some } L\right\},
$$




$$
w_{\infty}(f)=\left\{x \in s: \sup _{n} \frac{1}{n} \sum_{k=1}^{n} f\left(\left|x_{k}\right|\right)<\infty\right\},
$$

where $s$ is space of all complex sequences.

Later, Connor [12] extended his definition by replacing the Cesaro matrix with an arbitrary nonnegative matrix summability method $B=\left(b_{n_{k}}\right)$ as follow:

$$
\begin{aligned}
w & (B, f) \\
& =\left\{x \in s: \lim _{n \rightarrow \infty} \sum_{k=1}^{\infty} b_{n, k} f\left(\left|x_{k}-L\right|\right)=0 \text { for some } L\right\} .
\end{aligned}
$$

\section{Definitions and Notations}

Let $\sigma$ be a mapping of the positive integers into itself. A continuous linear functional $\varphi$ on $\ell_{\infty}$, the space of real bounded sequences, is said to be an invariant mean or a $\sigma$ mean, if and only if,

(1) $\phi(x) \geq 0$, for all sequences $x=\left(x_{n}\right)$ with $x_{n} \geq 0$ for all $n$;

(2) $\phi(e)=1$, where $e=(1,1,1, \ldots)$;

(3) $\phi\left(x_{\sigma(n)}\right)=\phi(x)$ for all $x \in \ell_{\infty}$.

The mappings $\phi$ are assumed to be one-to-one such that $\sigma^{m}(n) \neq n$ for all positive integers $n$ and $m$, where $\sigma^{m}(n)$ denotes the $m$ th iterate of the mapping $\sigma$ at $n$. Thus, $\phi$ extends the limit functional on $c$, the space of convergent sequences, in the sense that $\phi(x)=\lim x$, for all $x \in c$. In case $\sigma$ is translation mapping $\sigma(n)=n+1$, the $\sigma$ mean is often called a Banach limit and $V_{\sigma}$, the set of bounded sequences all of whose invariant means are equal, is the set of almost convergent sequences.

It can be shown that

$$
\begin{aligned}
V_{\sigma}=\{x & =\left(x_{n}\right): \lim _{n} t_{m n}(x)=L \\
& \text { uniformly in } m, L=\sigma-\lim x\},
\end{aligned}
$$

where,

$$
t_{m n}(x)=\frac{x_{m}+x_{\sigma(m)}+x_{\sigma^{2}(m)}+\cdots+x_{\sigma^{n}(m)}}{n+1} .
$$

Nuray and Savaş [13] defined the following sequence spaces by using a modulus function $f$ and a nonnegative regular matrix $B=\left(b_{n k}\right)$ :

$$
\begin{aligned}
& w_{0}\left(B_{\sigma}, f\right)=\left\{x \in s: \lim _{n \rightarrow \infty} \sum_{k=1}^{\infty} b_{n k} f\left(\left|x_{\sigma^{k}(m)}\right|\right)=0\right. \\
& \text { uniformly in } m\} \text {, } \\
& w\left(B_{\sigma}, f\right) \\
& =\left\{x \in s: \lim _{n \rightarrow \infty} \sum_{k=1}^{\infty} b_{n k} f\left(\left|x_{\sigma^{k}(m)}-L\right|\right)=0\right. \\
& \text { for some } L \text {, uniformly in } m\} \text {, } \\
& w_{\infty}\left(B_{\sigma}, f\right)=\left\{x \in s: \sup _{n, m} \sum_{k=1}^{\infty} b_{n k} f\left(\left|x_{\sigma^{k}(m)}\right|\right)<\infty\right\} .
\end{aligned}
$$

Definition 1 (see [14]). A set $E$ of positive integers is said to have uniform invariant density of zero if

$$
\lim _{n \rightarrow \infty} \frac{1}{n}\left|\left\{k \leq n: E \cap\left\{\sigma(m), \sigma^{2}(m), \ldots, \sigma^{k}(m)\right\}\right\}\right|=0
$$

uniformly in $m$.

By using uniform invariant density, the following definition was given.

Definition 2 (see [10]). A complex number sequence $x=\left(x_{k}\right)$ is said to be $\sigma$-statistically convergent to $L$ if, for every $\varepsilon>0$,

$$
\begin{array}{r}
\lim _{n} \frac{1}{n}\left|\left\{0 \leq k \leq n:\left|x_{\sigma^{k}(m)}-L\right| \geq \varepsilon\right\}\right|=0 \\
\text { uniformly in } m=1,2, \ldots
\end{array}
$$

This will be denoted by $S_{\sigma}-\lim x=L$ or $x_{k} \rightarrow L\left(S_{\sigma}\right)$.

Let $(X, \rho)$ be a metric space. For any point $x \in X$ and nonempty subset $A$ of $X$, we define the distance from $x$ to $A$ by

$$
d(x, A)=\inf _{a \in A} \rho(x, A) .
$$

The concept of Wijsman convergence was introduced by Wijsman [7] as follows.

Let $(X, \rho)$ be a metric space. For any nonempty closed subsets $A, A_{k} \subseteq X$, we say that the sequence $\left\{A_{k}\right\}$ is Wijsman convergent to $A$ if

$$
\lim _{k \rightarrow \infty} d\left(x, A_{k}\right)=d(x, A)
$$

for each $x \in X$. This will be denoted by $W-\lim A_{k}=A$.

Convergence concept for sequences of set had been studied by Beer [3], Aubin and Frankowska [4], and Baronti and 
Papini [5]. The concepts of Wijsman statistical convergence and Wijsman strong Cesaro summability were introduced by Nuray and Rhoades [6] as follows.

Let $(X, \rho)$ be a metric space. For any nonempty closed subsets $A, A_{k} \subseteq X$, the sequence $\left\{A_{k}\right\}$ is said to be Wijsman strongly Cesaro summable to $A$ if, for each $x \in X$,

$$
\lim _{n \rightarrow \infty} \frac{1}{n} \sum_{k=1}^{n}\left|d\left(x, A_{k}\right)-d(x, A)\right|=0 .
$$

Let $(X, \rho)$ be a metric space. For any nonempty closed subsets $A, A_{k} \subseteq X$, the sequence $\left\{A_{k}\right\}$ is said to be Wijsman statistically convergent to $A$ if, for $\varepsilon>0$ and each $x \in X$,

$$
\lim _{n \rightarrow \infty} \frac{1}{n}\left|\left\{k \leq n:\left|d\left(x, A_{k}\right)-d(x, A)\right| \geq \varepsilon\right\}\right|=0 .
$$

In this case we write $s t-\lim _{W} A_{k}=A$ or $A_{k} \rightarrow A(W S)$.

\section{Main Result}

The purpose of this paper is, by using a modulus function, to introduce and study new sequence spaces of sequences of sets. The following three definitions were given in [8].

Definition 3. Let $(X, \rho)$ be a metric space. For any nonempty closed subsets $A, A_{k} \subseteq X$, we say that the sequence $\left\{A_{k}\right\}$ is Wijsman invariant convergent to $A$, if, for each $x \in X$,

$$
\lim _{n \rightarrow \infty} \frac{1}{n} \sum_{k=1}^{n} d\left(x, A_{\sigma^{k}(m)}\right)=d(x, A)
$$

uniformly in $m$.

In this case, we write $A_{k} \rightarrow A\left(W V_{\sigma}\right)$ and the set of all Wijsman invariant convergent sequences of sets will be denoted $W V_{\sigma}$.

Definition 4. Let $(X, \rho)$ be a metric space. For any nonempty closed subsets $A, A_{k} \subseteq X$, we say that the sequence $\left\{A_{k}\right\}$ is Wijsman strongly invariant convergent to $A$, if for each $x \in$ $X$,

$$
\lim _{n \rightarrow \infty} \frac{1}{n} \sum_{k=1}^{n}\left|d\left(x, A_{\sigma^{k}(m)}\right)-d(x, A)\right|=0
$$

uniformly in $m$.

In this case, we write $A_{k} \rightarrow A\left(\left[W V_{\sigma}\right]\right)$ and the set of all Wijsman strongly invariant convergent sequences of sets will be denoted $\left[W V_{\sigma}\right]$.

Definition 5. Let $(X, \rho)$ be a metric space. For any nonempty closed subsets $A, A_{k} \subseteq X$, we say that the sequence $\left\{A_{k}\right\}$ is Wijsman invariant statistically convergent to $A$, if, for each $\varepsilon>0$ and for each $x \in X$,

$$
\lim _{n \rightarrow \infty} \frac{1}{n}\left|\left\{0 \leq k \leq n:\left|d\left(x, A_{\sigma^{k}(m)}\right)-d(x, A)\right| \geq \varepsilon\right\}\right|=0
$$

uniformly in $m$.
In this case, we write $A_{k} \rightarrow A\left(W S_{\sigma}\right)$ and the set of all Wijsman invariant statistically convergent sequences of sets will be denoted $W S_{\sigma}$.

Let $(X, \rho)$ be metric space. For any nonempty closed subsets $A, A_{k} \subseteq X$ and $x \in X$, we define the sequences of sets space $\left[W V_{\sigma}\right]_{\infty}$ as follows:

$$
\left[W V_{\sigma}\right]_{\infty}=\left\{\left\{A_{k}\right\} \sup _{m, n} \frac{1}{n} \sum_{k=1}^{n}\left|d\left(x, A_{\sigma^{k}(m)}\right)\right|<\infty\right\} .
$$

Now, by using a modulus function, we introduce the following new sequence spaces of sequences of sets.

Definition 6. Let $(X, \rho)$ be a metric space and $f$ be a modulus function. For any nonempty closed subsets $A, A_{k} \subseteq X$, we say that the sequence $\left\{A_{k}\right\}$ is Wijsman strongly invariant convergent to $A$ with respect to the modulus $f$, if, for each $x \in X$,

$$
\lim _{n \rightarrow \infty} \frac{1}{n} \sum_{k=1}^{n} f\left(\left|d\left(x, A_{\sigma^{k}(m)}\right)-d(x, A)\right|\right)=0
$$

uniformly in $m$.

In this case we write $A_{k} \rightarrow A\left(\left[W V_{\sigma}(f)\right]\right)$ and the set of all Wijsman strongly invariant convergent sequences of sets with respect to the modulus $f$ will be denoted $\left[W V_{\sigma}(f)\right]$.

Let $(X, \rho)$ be metric space and $f$ be a modulus function. For any nonempty closed subsets $A, A_{k} \subseteq X$ and $x \in X$, we define the sequences of sets space $\left[W V_{\sigma}(f)\right]_{\infty}$ as follows:

$\left[W V_{\sigma}(f)\right]_{\infty}=\left\{\left\{A_{k}\right\}: \sup _{m, n} \frac{1}{n} \sum_{k=1}^{n} f\left(\left|d\left(x, A_{\sigma^{k}(m)}\right)\right|\right)<\infty\right\}$.

If $f(x)=x$, then the spaces $\left[W V_{\sigma}(f)\right]$ and $\left[W V_{\sigma}(f)\right]_{\infty}$ reduce to $\left[W V_{\sigma}\right]$ and $\left[W V_{\sigma}\right]_{\infty}$, respectively.

Now we study the relation between $W S_{\sigma}$ and $\left[W V_{\sigma}(f)\right]$ convergence.

Theorem 7. Let $(X, \rho)$ be a metric space. For any nonempty closed subsets $A, A_{k} \subseteq X$. Then

(i) $A_{k} \rightarrow A\left(\left[W V_{\sigma}(f)\right]\right)$ implies $A_{k} \rightarrow A\left(W S_{\sigma}\right)$;

(ii) $f$ is bounded and $A_{k} \rightarrow A\left(W S_{\sigma}\right)$ implies $A_{k} \rightarrow$ $A\left(\left[W V_{\sigma}(f)\right]\right)$;

(iii) $W S_{\sigma}=\left[W V_{\sigma}(f)\right]$, if $f$ is bounded.

Proof. (i) Let $\varepsilon>0$ and $A_{k} \rightarrow A\left(\left[W V_{\sigma}(f)\right]\right)$. Then we can write

$$
\begin{aligned}
& \sum_{k=1}^{n} f\left(\left|d\left(x, A_{\sigma^{k}(m)}\right)-d(x, A)\right|\right) \\
& \quad \geq \sum_{\left|d\left(x, A_{\sigma^{k}(m)}\right)-d(x, A)\right| \geq \varepsilon}^{n} f\left(\left|d\left(x, A_{\sigma^{k}(m)}\right)-d(x, A)\right|\right) \\
& \geq \varepsilon \cdot\left|\left\{k \leq n:\left|d\left(x, A_{\sigma^{k}(m)}\right)-d(x, A)\right| \geq \varepsilon\right\}\right|,
\end{aligned}
$$

which yields the result. 
(ii) Suppose that $A_{k} \rightarrow A\left(W S_{\sigma}\right)$ and $f$ is bounded, for each $m \geq 1$, set

$$
G=\sup _{k, m} f\left(\left|d\left(x, A_{\sigma^{k}(m)}\right)\right|+|d(x, A)|\right) .
$$

Let $\varepsilon>0$ and select $N_{\varepsilon}$ such that

$$
\frac{1}{n}\left|\left\{k \leq n:\left|d\left(x, A_{\sigma^{k}(m)}\right)-d(x, A)\right| \geq \frac{\varepsilon}{2}\right\}\right|<\frac{\varepsilon}{2 G},
$$

for all $m$ and $n>N_{\varepsilon}$, and set $L(n, m, x)=\{k \leq n$ : $\left.\left|d\left(x, A_{\sigma^{k}(m)}\right)-d(x, A)\right| \geq \varepsilon / 2\right\}$. Now, for all $m$ and $n>N_{\varepsilon}$, we have that

$$
\begin{aligned}
\sum_{k=1}^{n} f & \left(\left|d\left(x, A_{\sigma^{k}(m)}\right)-d(x, A)\right|\right) \\
= & \sum_{k \in L(n, m, x)} f\left(\left|d\left(x, A_{\sigma^{k}(m)}\right)-d(x, A)\right|\right) \\
& +\sum_{k \notin L(n, m, x)} f\left(\left|d\left(x, A_{\sigma^{k}(m)}\right)-d(x, A)\right|\right) \\
< & \frac{1}{n}\left(n \frac{\varepsilon}{2 G}\right) G+\frac{1}{n}\left(\frac{\varepsilon}{2}\right) n=\frac{\varepsilon}{2}+\frac{\varepsilon}{2}=\varepsilon .
\end{aligned}
$$

Hence, $\left\{A_{k}\right\}$ is strongly invariant convergent to $A$ with respect to the modulus function $f$.

(iii) This is an immediate consequence of (i) and (ii).

This completes the proof of the theorem.

Theorem 8. Let $f$ be a modulus function. Then $\left[W V_{\sigma}(f)\right] \subset$ $\left[W V_{\sigma}(f)\right]_{\infty}$.

Proof. Suppose that, $\left\{A_{k}\right\} \in\left[W V_{\sigma}(f)\right]$. Then we can write

$$
\begin{aligned}
& \frac{1}{n} \sum_{k=1}^{n} f\left(\left|d\left(x, A_{\sigma^{k}(m)}\right)\right|\right) \\
& \quad=\frac{1}{n} \sum_{k=1}^{n} f\left(\left|d\left(x, A_{\sigma^{k}(m)}\right)-d(x, A)+d(x, A)\right|\right) \\
& \quad \leq \frac{1}{n} \sum_{k=1}^{n} f\left(\left|d\left(x, A_{\sigma^{k}(m)}\right)-d(x, A)\right|\right)+\frac{1}{n} \sum_{k=1}^{n} f(|d(x, A)|) \\
& \quad \leq \frac{1}{n} \sum_{k=1}^{n} f\left(\left|d\left(x, A_{\sigma^{k}(m)}\right)-d(x, A)\right|\right)+M \frac{1}{n} \sum_{k=1}^{n} f(1),
\end{aligned}
$$

where $M$ is an integer such that $d(x, A)<M$. Therefore, $\left\{A_{k}\right\} \in\left[W V_{\sigma}(f)\right]_{\infty}$.

Theorem 9. If $f$ is a modulus function and $\left\{A_{k}\right\}$ is strongly invariant convergent to $A$, then $\left\{A_{k}\right\}$ is strongly invariant convergent to $A$ with respect to the modulus $f$; that is, $\left[W V_{\sigma}\right] \subset$ $\left[W V_{\sigma}(f)\right]$.
Proof. Let $\left\{A_{k}\right\} \in\left[W V_{\sigma}\right]$. Then we can write

$$
S_{n m}=\frac{1}{n} \sum_{k=1}^{n}\left|d\left(x, A_{\sigma^{k}(m)}\right)-d(x, A)\right| \longrightarrow 0 \quad(n \longrightarrow \infty)
$$

uniformly in $m$.

Let $\varepsilon>0$ and choose $\delta$ with $0<\delta<1$ such that $f(t)<\varepsilon$ for $0 \leq t \leq \delta$. Write $a_{k m}=\left|d\left(x, A_{\sigma^{k}(m)}\right)-d(x, A)\right|$,

$$
\sum_{k=1}^{n} f\left(a_{k m}\right)=\Sigma_{1}+\Sigma_{2}
$$

where the first summation is over $a_{k m} \leq \delta$ and second over $a_{k m}>\delta$. Then $\Sigma_{1} \leq \varepsilon n$ and, for $a_{k m}>\delta$,

$$
f\left(a_{k m}\right)<\frac{a_{k m}}{\delta}<1+\left[\frac{a_{k m}}{\delta}\right]
$$

where $[z]$ denotes the integer part of $z$. By definition modulus function, we have, for $a_{k m}>\delta$,

$$
f\left(a_{k m}\right) \leq\left(1+\left[\frac{a_{k m}}{\delta}\right]\right) f(1) \leq 2 f(1) \frac{a_{k m}}{\delta} .
$$

Hence, $\Sigma_{2} \leq 2 f(1)\left(a_{k m} / \delta\right)$, which together with $\Sigma_{1} \leq \varepsilon n$ yields $\left[W V_{\sigma}\right] \subset\left[W V_{\sigma}(f)\right]$.

Lemma 10 (see [15]). Let $f$ be a modulus function. Let $\alpha>0$ be given constant. Then, there is a constant $c>0$ such that $f(x)>c x(0<x<\alpha)$.

Theorem 11. Let $\left\{A_{k}\right\}$ be a bounded sequence and $f$ be a modulus function. Then $\left\{A_{k}\right\}$ is Wijsman strongly invariant convergent to $A$ with respect to modulus $f$ if and only if $\left\{A_{k}\right\}$ is Wijsman strongly invariant convergent to A; that is,

$$
\left[W V_{\sigma}(f)\right] \cap L_{\infty}=\left[W V_{\sigma}\right]
$$

where $L_{\infty}$ denotes the set of bounded sequences of sets.

Proof. The proof of the theorem follows from Theorem 9 and Lemma 10.

\section{Conflict of Interests}

The authors declare that there is no conflict of interests regarding the publication of this paper.

\section{References}

[1] H. Fast, "Sur la convergence statistique," Colloquium Mathematicae, vol. 2, no. 3-4, pp. 241-244, 1951.

[2] T. Šalát, "On statistically convergent sequences of real numbers," Mathematica Slovaca, vol. 30, no. 2, pp. 139-150, 1980.

[3] G. Beer, "Convergence of continuous linear functionals and their level sets," Archiv der Mathematik, vol. 52, no. 5, pp. 482491, 1989.

[4] J. P. Aubin and H. Frankowska, Set Valued Analysis, Birkhäuser, Boston, Mass, USA, 1990. 
[5] M. Baronti and P. L. Papini, "Convergence of sequences of sets," in Methods of Functional Analysis in Approximation Theory, vol. 76, pp. 135-155, Birkhäuser, Basel, Switzerland, 1986.

[6] F. Nuray and B. E. Rhoades, "Statistical convergence of sequences of sets," Fasciculi Mathematici, no. 49, pp. 87-99, 2012.

[7] U. Ulusu and F. Nuray, "Lacunary statistical convergence sequences of sets," Progress in Applied Mathematics, vol. 4, no. 2, pp. 99-109, 2012.

[8] N. Pancaroglu and F. Nuray, "On invariant statistically convergence and lacu-nary invariant statistically convergence of sequences of sets," Progress in Applied Mathematics, vol. 5, no. 2, pp. 23-29, 2013.

[9] H. Nakano, "Concave modulars," Journal of the Mathematical Society of Japan, vol. 5, no. 1, pp. 29-49, 1953.

[10] W. H. Ruckle, "FK spaces in which the sequence of coordinate vectors is bounded," Canadian Journal of Mathematics, vol. 25, pp. 973-978, 1973.

[11] I. J. Maddox, "Sequence spaces defined by a modulus," Mathematical Proceedings of the Cambridge Philosophical Society, vol. 100, no. 1, pp. 161-166, 1986.

[12] J. Connor, "On strong matrix summability with respect to a modulus and statistical convergence," Canadian Mathematical Bulletin, vol. 32, no. 2, pp. 194-198, 1989.

[13] F. Nuray and E. Savaş, "Some new sequence spaces defined by a modulus function," Indian Journal of Pure and Applied Mathematics, vol. 24, no. 11, pp. 657-663, 1993.

[14] E. Savaş, "Strongly $\sigma$-convergent sequences," Bulletin of the Calcutta Mathematical Society, vol. 81, no. 4, pp. 295-300, 1989.

[15] E. Savaş, "On strong almost $A$-summability with respect to a modulus and statistical convergence," Indian Journal of Pure and Applied Mathematics, vol. 23, no. 3, pp. 217-222, 1992. 


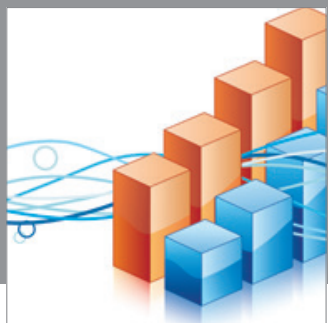

Advances in

Operations Research

mansans

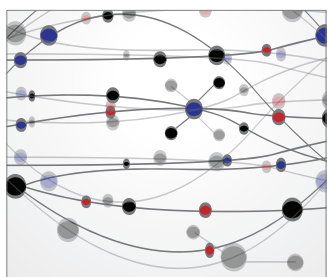

The Scientific World Journal
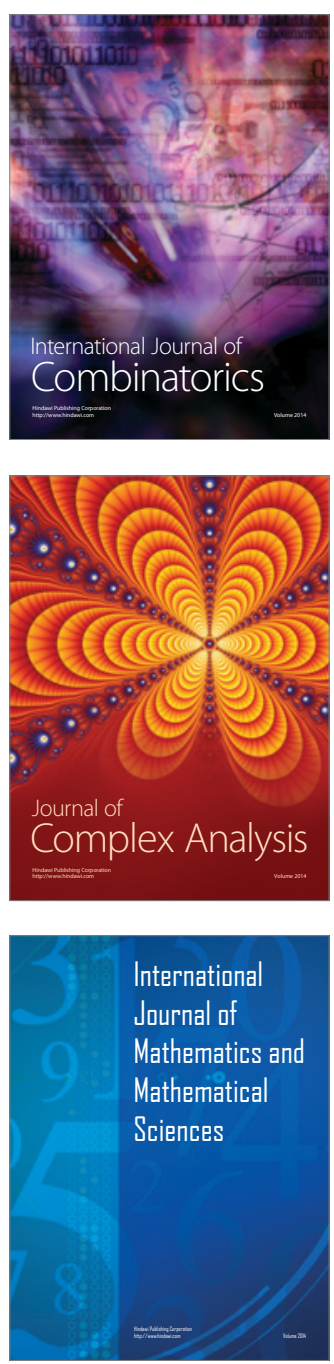
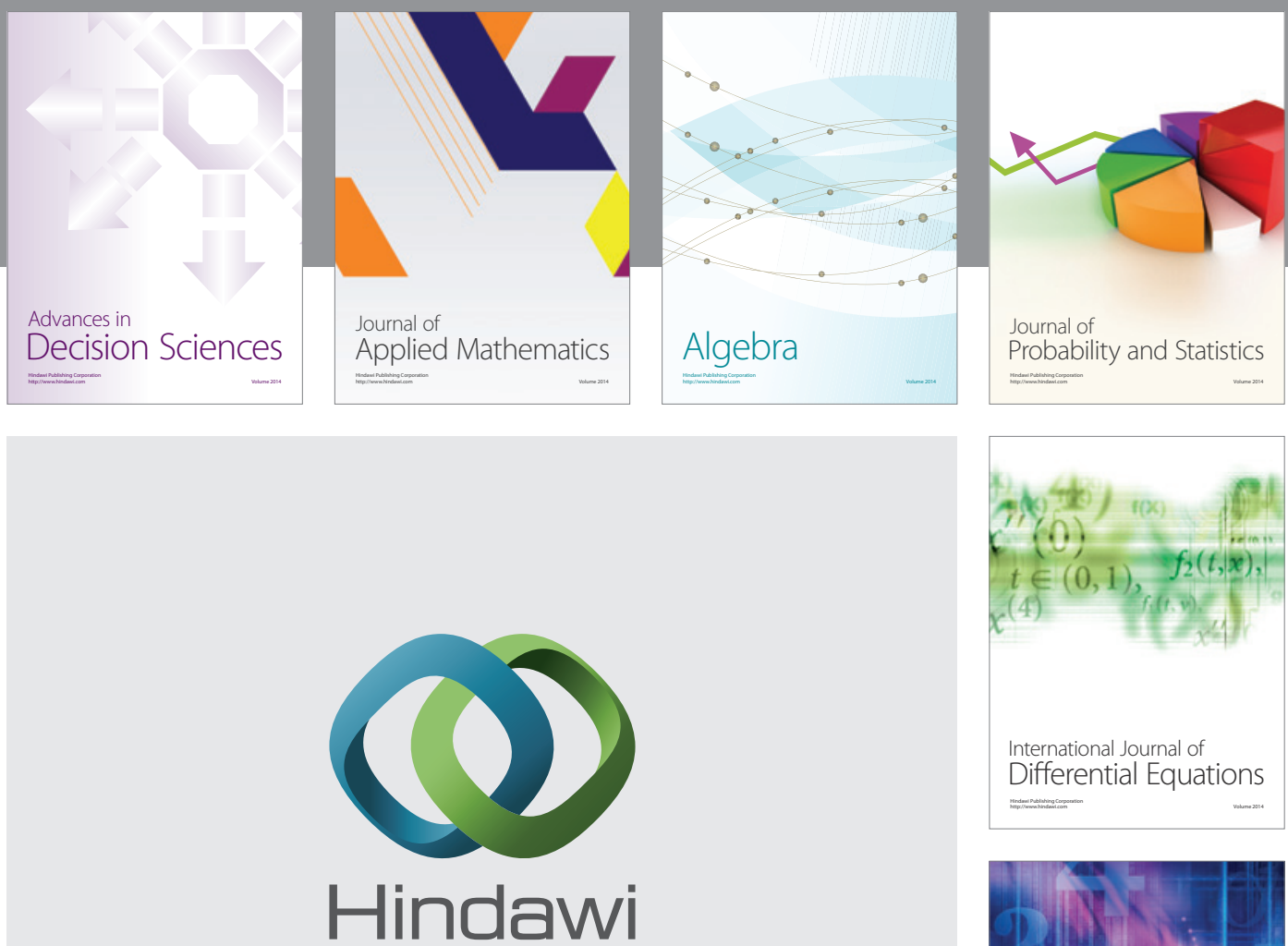

Submit your manuscripts at http://www.hindawi.com
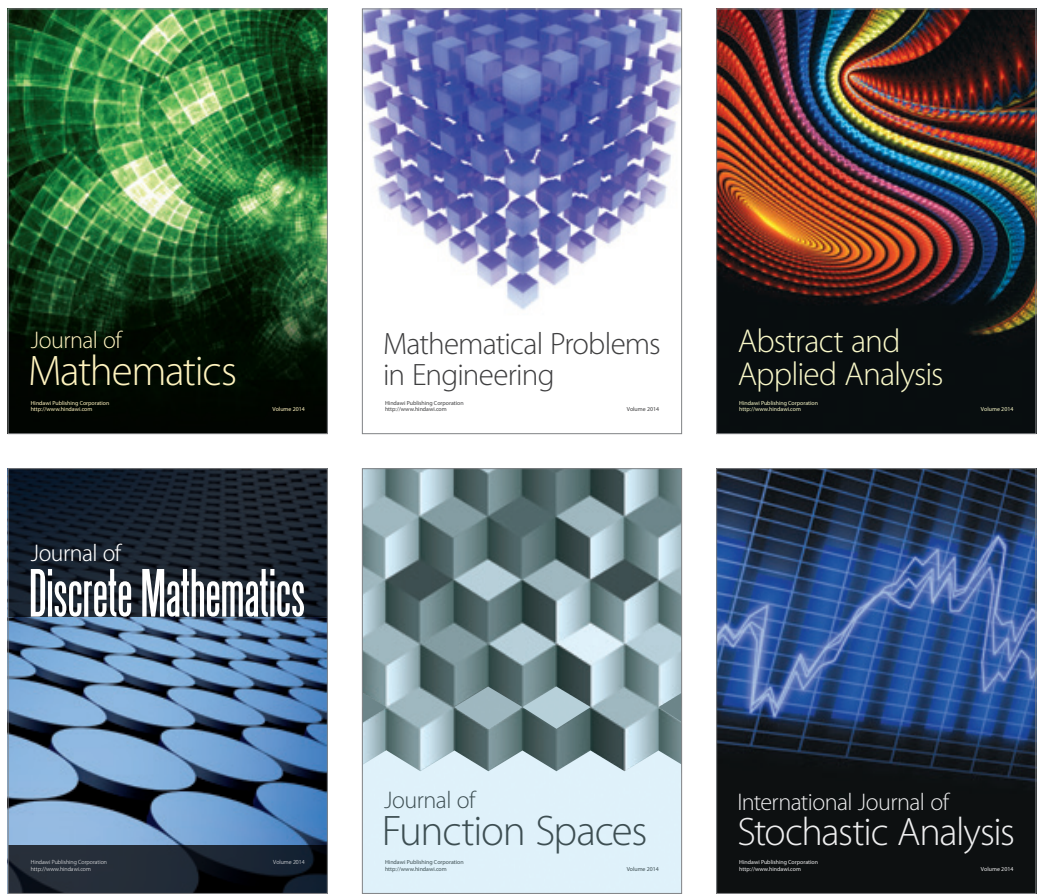

Journal of

Function Spaces

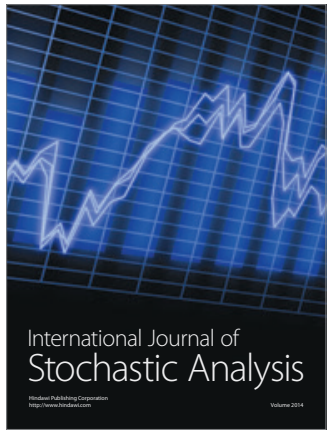

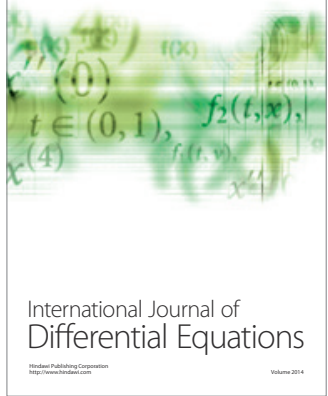
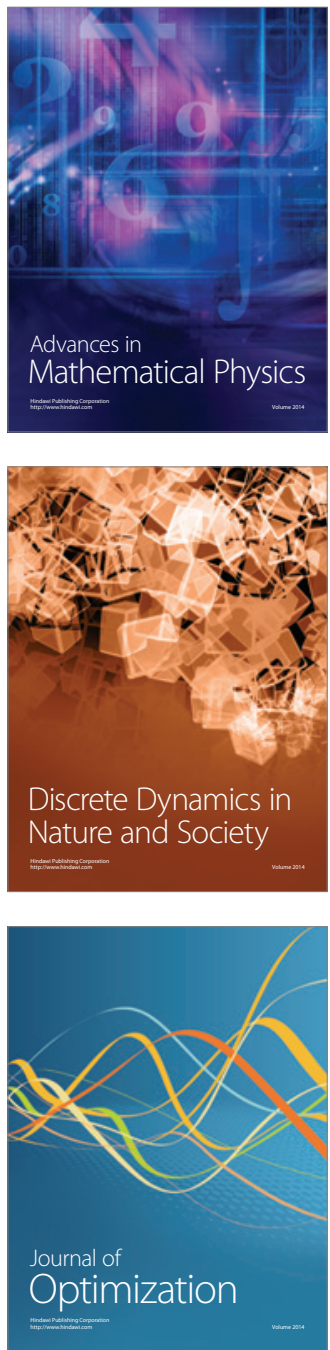